\title{
Дисконтирование денежных потоков в оценке инвестиционной привлекательности бизнеса в АTP
}

\author{
Дмитрий Скалкин, Елена Селезнева ${ }^{*}$, Светлана Ракутько
}

Дальневосточный федеральный университет, г. Владивосток, Россия

\author{
Информация о статье \\ Поступила в редакичию: \\ 10.03.2020 \\ Принята \\ к опубликованию: \\ 24.06.2020 \\ УДК 330.3 \\ JEL E4, E5, P33
}

\section{Ключевые слова:}

Азиатско-тихоокеанский регион, инвестиционный климат, дисконтирование денежных потоков, инвестиционная привлекательность, инвестиции, оценка стоимости бизнеса, оценочные стандарты России

\section{Keywords:}

Asia-Pacific region (APR), investment climate, discounting cash flows (DCF), investment attractiveness, investment, business valuation, evaluation Standards of Russia

\begin{abstract}
Аннотация
В статье авторы рассматривают перспективу применения метода дисконтирования денежных потоков (далее ДДП) в оценке привлекательности российского бизнеса для предпринимателей Азиатско-Тихоокеанского региона (далее - АТР). Обозначена роль АТР в улучшении национального инвестиционного климата, перспективы взаимодействия России с другими странами региона с ичелью повьшения инвестиционной привлекательности российского бизнеса. Дано обобщающее определение понятия «инвестиционная привлекательность» с точки зрения инвесторов и авторское понимание данной категории. Показана высокая чувствительность метода ДДП к прогнозируемым ценам, представлень достоинства, недостатки и дискуссионные вопросы по его применению в оченке привлекательности российского бизнеса. Рассмотрены преимущества роста качества инвестиционной привлекательности страны. Обозначена проблема оченочных стандартов России. Предложень методы по совериенствованию оценки привлекательности бизнеса с использованием метода ДДП. Применение данного метода, хоть и имеет свои недостатки, особенно целесообразно, по мнению авторов, когда оценивается инвестиционная привлекательность предприятий, длительное время успешно функционирующих на рынке и находящихся в стадии стабильного экономического развития.
\end{abstract}

Discounting Cash Flows in Assessing the Business Investment Attractiveness in the Asia-Pacific Region

Dmitriy Skalkin, Elena Selezneva, Svetlana Rakutko

\section{Abstract}

The article describes the method of discounting cash flows (DCF) in assessing the business attractiveness. The information related to the use of this parameter in Russia has been analyzed. The role of the Asia-Pacific region in improving

\footnotetext{
* Автор для связи: selezneva.eyи@dvfu.ru

DOI: https://dx.doi.org/10.24866/2311-2271/2020-2/94-106
} 
the national investment climate, the prospects for Russia interaction in the Asia-Pacific region aimed at increasing the investment attractiveness of the country's own business have been outlined. The generalized definition of the "investment attractiveness" concept has been shown from the investors' point of view. High sensitivity of the cash flow discounting method to the predicted prices has been demonstrated. The advantages, disadvantages and debating questions on application of cash flow discounting method in estimating the attractiveness of the Russian business have been described. The advantages of increasing the quality of the country's investment attractiveness have been considered. The problem of the evaluation Standards of Russia has been specified. Methods to improve the quality of the business attractiveness assessing using the DCF methods have been proposed. According to the authors, the application of this method is especially appropriate, when assessing the investment attractiveness of enterprises that have been successfully operating on the market for a long time and are in the stage of stable economic development.

\section{Введение}

Привлечение инвестиционных потоков - одна из основных проблем социально-экономического развития стран, регионов, отраслей и хозяйствующих субъектов в существующих экономических реалиях. В связи с появлением новых информационных технологий ускоряются рыночные процессы и повышается динамичность рынка и инвестиционные риски. Перечисленные факторы оказывают значительное влияние на решения инвесторов в вопросе оценки инвестиционного климата и инвестиционной привлекательности потенциальных объектов инвестирования.

На сегодняшний день инвестиционный климат Российской Федерации не располагает к притоку денежных средств. Тем не менее, важным условием для эффективного ведения национальной экономики остается привлечение инвестиционных потоков на внутренний рынок. Россия нуждается в расширении инвестиционной базы для развития инновационной деятельности и укрепления экономического потенциала национальной экономики. Одним из наиболее очевидных методов решения данной проблемы является интенсивная интеграция России в АТР и расширение взаимовыгодного сотрудничества со странами, входящими в его состав.

Страны Азиатско-тихоокеанского региона - это государства с высокими темпами развития экономики, со значительным по численности населением и обширными территориями, на которых расположено огромное количество промышленных предприятий, как правило, осуществляющих производство наукоемкой продукции. Страны АТР интересны для России и по причине высокого уровня капитализации многих из них, возможности реализации совместных проектов и нейтральной или близкой позиции по наиболее значимым проблемам международных отношений. Очевидна заинтересованность российских предпринимателей к установлению долговременных деловых контактов в АТР. При этом потенциал взаимодействия в торговоэкономической сфере многократно превышает нынешние объемы делового сотрудничества с региональными партнерами.

Одна из существенных причин невысоких темпов экономического роста Сибири, Дальнего Востока и Забайкалья заключается в том, что ранее не было уделено достаточное внимание потенциалу сотрудничества с соседними странами в решении стоящих перед Россией задач, особенно в области наращивания инвестиционного потенциала. 
Таким образом, полноценное включение России и, прежде всего, Дальнего Востока и Сибири в систему мирохозяйственных связей АТР представляет собой одно из ключевых условий социально-экономического развития страны и укрепления инвестиционной привлекательности российского бизнеса. Азиатско-Тихоокеанское направление должно стать основным вектором стратегии взаимодействия российского Дальнего Востока и Сибири с мировым хозяйством.

Российские предпринимательские структуры заинтересованы в увеличении рентабельности и продуктивности, конкурентоспособности и финансовой независимости. Данные показатели имеют прямую корреляционную зависимость с существующим уровнем инвестиционной активности и инвестиционной привлекательности бизнеса. В связи с тем, что любые инвестиции влекут за собой риски, оценка инвестиционной привлекательности компании - один из эффективных методов, направленный на их снижение. Для этого используется множество показателей и методов, в том числе и ДДП. Использование данного метода для отечественных компаний является наиболее приемлемым, так как позволяет влиять на мнение зарубежных инвесторов из стран АТР об инвестиционной привлекательности российских компаний. Это, в первую очередь, связано с тем, что акции большинства компаний России и стран АТР не оборачиваются на биржах, от чего оценка инвестиционной привлекательности затруднительна по сравнению с компаниями, участвующими в биржевых торгах. В связи с этим применение метода ДДП помогает оценить возможные будущие экономические выгоды от инвестиционной деятельности, как стран АТР, инвестирующих в российское предпринимательство, так и наоборот.

Высокий уровень инвестиционной привлекательности формирует и укрепляет видимые и скрытые преимущества компании перед конкурентами [1] и является фактором результативности её деятельности.

Поскольку инвестирование подразумевает получение будущих экономических выгод, то для того, чтобы принять решение об инвестировании, необходимо соотнести ожидания от будущих поступлений с нынешними затратами на капитальные вложения. Вопросы инвестирования и инвестиционной привлекательности носят прикладной характер, так как их решение обеспечивает не только результативность деятельности отдельной компании, но экономический рост в целом.

На сегодняшний день остаётся не раскрытым множество теоретических особенностей оценки инвестиционной привлекательности предприятий, которые необходимо изучить и апробировать. До сих пор среди учёных нет общего мнения насчет рациональности применения данного метода для компаний, генерирующих отрицательные денежные потоки. Дискуссионный характер проблемы подтверждает отсутствие общепризнанного определения понятия «инвестиционная привлекательность предприятия». Также не определена система признаков для научно признанной классификации инвестиционной привлекательности и чёткого определения места данного явления на инвестиционном рынке страны. Немаловажным является то, что отсутствует единая методология проведения оценки привлекательности бизнеса при помощи метода ДДП. Специалистами в данной области определены отдельные важ- 
ные положения и позиции, которые обязательны к анализу, однако нет установленного алгоритма действий и системы конкретных показателей. Перечисленные составляющие не выделены как обязательные и оставлены на усмотрение эксперта, отчего результаты анализа привлекательности одной и той же компании могут значительно отличаться в зависимости от действий экспертов, проводивших анализ.

Одной из наиболее значимых проблем на сегодняшний день, по нашему мнению, является необходимость создания единой аналитической системы качественных и количественных показателей, пригодной для комплексной оценки инвестиционной привлекательности компании. В настоящее время оценка инвестиционной привлекательности организаций проводится, как правило, с использованием разных групп показателей и зачастую на основе несовершенных методик, что оказывает влияние на достоверность результатов.

Разнообразные методические материалы, представленные в специальной литературе, сети Интернет и периодической печати, касающиеся оценки инвестиционной привлекательности бизнеса, обычно предполагают использование в основном количественных факторов. Данный факт не позволяет учитывать полную совокупность характеристик, необходимых для качественной оценки инвестиционной привлекательности бизнеса, что ведёт к формулированию недостоверных выводов.

В настоящее время применение метода ДДП в оценке привлекательности бизнеса является достаточно частым явлением, однако целесообразность его применения стоит под вопросом. Возможно, причина в том, что для качественного применения этого метода необходимо учитывать и анализировать множество количественных и качественных факторов, объём которых иногда превышает количество факторов, необходимых для оценки инвестиционной привлекательности бизнеса. Также существует значительное количество вопросов в данном направлении, которые не имеют однозначных ответов в научной среде. В связи с этим применение метода ДДП в оценке инвестиционной привлекательности бизнеса является методом, полезность и адекватность использования которого необходимо пересмотреть не только в теоретическом аспекте, но и в практическом применении.

Целью статьи является выявление объективности и адекватности использования метода ДДП в оценке инвестиционной привлекательности российского бизнеса для стран АТР. Объект - методика оценки инвестиционной привлекательности. Предмет - дисконтирование денежных потоков в оценке привлекательности российского бизнеса для стран АТР.

\section{Теоретическая основа исследования}

Использование метода ДДП в оценке инвестиционной привлекательности каждой отдельной организации является обширным и многоэтапным процессом, включающим в себя разнообразные экономические аббревиатуры и понятия. Вполне очевидно, что наиболее важными для заказчика являются понятия «инвестиции» и «инвестиционная привлекательность». С целью получения информации, достоверной и понятной для всех участников процесса оценки, необходимо определиться с толкованием данных понятий.

Инвестиции - это вложение капитала для получения в перспективе вы- 
год определенного характера, выражение которых возможно [2]:

- в получении прибыли (снижение себестоимости, расширении производства, производстве новой продукции);

- в социальном эффекте (увеличении рабочих мест, гарантии стабильности и охраны труда, улучшении экологических условий хозяйствования).

Для того, чтобы у инвестора появилось желание инвестировать средства в то или иное предприятие, необходимо, чтобы последнее обладало инвестиционной привлекательностью, которую инвестор определяет путем анализа множества факторов.

Следует отметить, что «инвестиционная привлекательность», являющаяся крайне важной для понимания многих вопросов инвестиционной деятельности, до настоящего времени не нашла единого толкования среди исследователей, в частности, в экономической сфере. Дискуссионность данного термина дополняется многообразием подходов к определению категории «финансовое состояние предприятия».

Проанализируем мнения специалистов-исследователей по вопросам сущности инвестиционной привлекательности. Так, Г. Бирман и С. Шмидт определяют инвестиционную привлекательность как совокупность показателей, характеризующих финансовое состояние предприятия [3]. И.А. Бланк указывает на ее непосредственную связь со стадиями жизненного цикла предприятия [4]. А.Б. Крутик, Д.А. Ендовицкий, и Г. Никольская определяют инвестиционную привлекательность как общую характеристику всех недостатков и преимуществ рассматриваемого предприятия $[5,6]$.

По мнению М.Н. Крейнина, инвестиционная привлекательность состоит из множественности фундаментальных и фондовых показателей объекта инвестирования [7]. С. Бухдрукер считает ее интегральной характеристикой объекта с позиций доходности инвестиций, перспективности развития и существующего уровня инвестиционных рисков [8]. С.С. Донцов и В.В. Бочаров соотносят инвестиционную привлекательность с надежностью выпускаемых предприятием товаров/услуг [9, 10]. С.П. Конторович признает инвестиционную привлекательность как систему экономических отношений между хозяйствующими субъектами относительно эффективности развития бизнеса и поддержания его конкурентоспособности, в которые входят формальные и неформальные показатели аспектов деятельности предприятия [11]. Расчет формальных показателей базируется на данных финансовой отчетности. Л.Ф. Мухаметшина считает инвестиционную привлекательность самостоятельной экономической единицей, являющейся совокупной характеристикой инвестиционного потенциала предприятия, отражающей эффективное использование активов предприятия, существующий уровень инвестиционных рисков.

Представляется, что приведенные определения не лишены некоторых недостатков, не позволяющих признать их совершенными. Авторы статьи считают, что данные определения все же не могут быть рассмотрены как абсолютно точно отображающие весь комплекс факторов, значимых для характеристики инвестиционной привлекательности, в силу чего, видится необходимым формулирование данного понятия с точки зрения инвесторов. Мы предлагаем, учитывая интересы инвесторов, определить инвестиционную привлекательность, как систему внешних и внутренних факторов, обладающих количе- 
ственным и качественным составом, характеризующих финансовоэкономическое состояние предприятия, уровень его инвестиционных рисков, и отражающих платежеспособный спрос предприятия на инвестиции [12].

Для дальнейшего исследования уточним понятие дисконтирования денежного потока. Денежный поток предприятия - совокупность распределенных во времени поступлений и выплат денежных средств, генерируемых его хозяйственной деятельностью.

Понятие «денежный поток предприятия» может быть охарактеризовано как агрегированное явление, поскольку в него входит множество различных потоков, позволяющих производить обслуживание хозяйственной деятельности. Анализировать денежные потоки и проводить расчетные операции следует с учетом фактора времени, для чего и используется метод ДДП.

Указанный метод принято считать наиболее приемлемым с точки зрения инвестиционных мотивов, так как при инвестировании происходит приобретение не материальных объектов предприятия (зданий, оборудования, ценностей и пр.), а тех доходов, которые планируются в получении в будущем, имея при этом цель получения финансовой выгоды. Если анализировать предприятия, задействованные в любой сфере экономической деятельности с позиции рассматриваемого метода, каждым из них производится лишь такой вид продукции, как деньги. Метод ДДП базируется на том, что критерием измерения прогнозируемых доходов от бизнеса, учитываемых при определении рыночной стоимости предприятия, выступают не те прибыли, которые ожидаются от деятельности предприятия, а денежные потоки. Однако, в научной среде спорным остаётся вопрос относительно применения данного метода в случае постоянного генерирования компанией отрицательных денежных потоков. Так, С.А. Смоляк предлагает продисконтировать отрицательные денежные потоки деятельности организации, используя при этом наиболее низкую ставку, если она является стабильной [13]. О.П. Масленникова пишет, что необходимо применять одинаковую ставку дисконтирования для любых денежных потоков (положительных или отрицательных), если сохраняется один уровень риска [14].

Е.П. Фомин отрицает возможность использования метода дисконтирования денежного потока при отрицательных денежных потоках организации. Согласно его мнению, инвестиции в организации с отрицательным денежным потоком являются более рисковыми, вследствие чего такие инвестиции воспринимается как более ценные (чем больше риск, тем больше должна быть прибыль), что противоречит гипотезе об инвесторе, избегающем риска [15].

Таким образом, по мнению авторов, использование метода ДДП возможно при оценке деятельности любого предприятия, вне зависимости от его финансового состояния, положения на рынке, отраслевой принадлежности. В то же время, в некоторых ситуациях применение рассматриваемого метода наиболее оправдано, в частности, когда оценивается инвестиционная привлекательность предприятий, достаточно длительное время существующих на рынке, имеющих прибыльную хозяйственную деятельность, а также предприятий, находящихся в стадии роста или стабильного экономического развития. В отношении убыточных предприятий применение рассматриваемого 
метода не рекомендуется в связи с тем, что оценка отрицательных денежных потоков не имеет экономического смысла в разрезе инвестиций, так как полученные результаты расчетов тоже будут отрицательны, и можно будет рассматривать лишь их модульные значения, что усилит условность оценивания.

С определенной долей осмотрительности следует подходить к применению рассматриваемого метода в том случае, когда осуществляется оценка деятельности недавно созданных предприятий, даже если первоначальные результаты их деятельности свидетельствуют о том, что они являются перспективными. В данном случае все же невозможно произвести ретроспективный анализ за длительный период и определить системность и регулярность получения прибылей.

Следует отметить, что вопрос применения метода ДДП в учетной деятельности стран АТР особенно актуален [16]. Это, в первую очередь, связано с информативностью отчета о движении денежных средств, и с тем, что для большинства азиатских стран, которые является крупнейшими торговыми партнерами России, является обязательным включение этой формы отчетности в бухгалтерскую (финансовую) отчетность как отдельного документа. В российской учетной практике Отчет о движении денежных средств (далее ОДДС) является приложением к бухгалтерскому балансу и его составление не обязательно для организаций, имеющих право на составление упрощённой отчетности. Таким образом, состав и функционал бухгалтерской (финансовой) отчетности многих стран АТР напрямую связаны с использованием метода ДДП в оценке привлекательности бизнеса. Факт того, что в российской учетной практике информативность ОДДС недостаточна и составление отчета не обязательно для целого сегмента бизнес сообщества, оказывает влияние на достоверность и прозрачность российской бухгалтерской отчетности в понимании потенциальных контрагентов и инвесторов. Поскольку в качественной оценке инвестиционной привлекательности бизнес партнёров заинтересованы все участники международного сотрудничества, России необходимо совершенствовать учетную практику, в том числе развивать уровень применения метода ДДП, используя опыт иностранных коллег. Такие меры помогут обеспечить инвестиционную привлекательность российскому бизнесу и будут способствовать повышению инвестиционной активности в Азиатско-Тихоокеанском регионе.

Таким образом, авторы считают, что метод ДДП является полезным инструментом для оценки стоимости бизнеса не только в рамках внутригосударственного использования, но и в международном бизнесе. Несмотря на то, что действительно качественное применение данного метода является трудоёмким и дорогостоящим, его аналитичность и вариативность помогают определить дисконтированную (приведенную) стоимость компании. Однако значительная часть проанализированных теоретических данных не даёт ответа на вопрос о том, как использовать данный метод в «неоднозначных» ситуациях, например при недавно начатой или убыточной деятельности. Также, недостаточное внимание уделяется фактору времени в оценке дисконтированных денежных потоков, что является немаловажным фактором и условием применения данного метода. Мнение относительно объективности и адекватности применения ДДП остается дискуссионным. 


\section{Методология исследования}

В процессе проведения исследования были проанализированы и использованы российские и международные нормативные документы в области регулирования бухгалтерского учета и аудита, нормативные акты Российской Федерации, единые стандарты профессиональной практики оценки США, труды современных зарубежных и российских ученых в данной области.

Методологическую основу исследования составили общенаучные подходы: комплексный, системный, логический. Их использование обосновано тем, что процесс оценки инвестиционной привлекательности представляет собой большое количество отдельных операций, являющихся частью единого целого, и влияние которых на результат было изучено и проанализировано. Также, авторы применили проектный метод для корректировки задач в процессе исследования и получения результатов [17].

Применение общенаучных методов научного познания (анализа и синтеза, систематизации и классификации, индукции и дедукции, сравнения и обобщения) было необходимо авторам для того, что разделить результат оценки инвестиционной привлекательности, выделить наиболее значимые его составляющие и объединить только их. Благодаря применению данных методов стало возможным разделение факторов на исчислимые и неисчислимые, количественные и качественные. В результате сделано сравнение влияния тех или иных составляющих на общий результат оценки привлекательности, выделены составляющие значительного влияния.

На начальном этапе исследования авторы использовали Excel систему для расчета дисконтированной стоимости акций для ежегодной оценки. В систему вошли не только количественные, но и качественные показатели, значения которых невозможно объективно определить (для составления качественного субъективного мнения необходимо быть экспертом). Авторы статьи путём подстановок определили, что влияние качественных значений на итоговый результат не является основополагающим и, что изменения данных значений в достаточно широком диапазоне незначительно влияет на конечный результативный показатель. Поэтому было принято решение не вносить поправки в определённые экспертами числовые выражения качественных показателей.

На следующем этапе авторы сравнили прогнозные значения, поддающиеся числовому выражению, с фактическими значениями. Практически все прогнозные значения оказались заниженными, таким образом, прогнозная стоимость предприятия даже на первый год оценки значительно отличалась от фактической. В связи с тем, что метод ДДП при прогнозировании стоимости использует значения предыдущих периодов для прогноза значений будущих периодов, то с каждым следующим годом значительно увеличивался диапазон ошибки.

Далее авторы сравнили фактическую стоимость акций с их прогнозной стоимостью, что дало возможность сделать вывод о качестве использования метода ДДП. Затем в исходную систему также были перенесены фактические значения для проверки качества модели с целью сравнения суммарного выражения прогнозной стоимости акций с суммарным фактическим значением их стоимости. В данном случае, модель показала минимальные отклонения 
расчетной стоимости от фактической, что позволило сделать вывод о значительном влиянии на результат применения метода ДДП для прогнозных либо фактических значений, особенно на поздних этапах прогнозирования.

\section{Обсуждение полученных результатов}

В американских Единых стандартах профессиональной практики оценки касательно метода ДДП отмечается [18], что «вследствие взаимно усиливающихся эффектов даже небольшие ошибки входных величин могут разрастись и привести к неправдоподобным результатам», стоит отметить, что выводы о качестве метода ДДП, который способен показывать недостоверные результаты, не делаются.

Чистый денежный поток в подавляющем большинстве случаев представляет собой часть выручки, так, в черной металлургии чистый денежный поток на собственный капитал составляет от 8 до $25 \%$ выручки от основного вида деятельности. При этом условно-постоянные затраты, участвующие в формировании себестоимости продукции, составляют оставшуюся основную часть - от 92 до 75\% разницы между выручкой и чистым денежным потоком. В связи с этим ошибка в прогнозируемой стоимости выпускаемой продукции, a, значит, и в прогнозируемой выручке, например, на 20\% приводит к ошибке в прогнозируемом чистом денежном потоке. В настоящее время уменьшение ошибки в прогнозе хотя бы до 10\% по выручке за первый прогнозный год является крайне сложной задачей. Рассмотрев прогнозные значения выручки из годовых бизнес-планов организаций, составленных в прошлые периоды, и сравнив их с показателями фактически полученной выручки, можно прийти к выводу, что разница составит от 10 до $30 \%$.

Таким образом, чтобы достичь точности в оценке стоимости бизнеса предприятия в 20\% оценщик должен уметь прогнозировать стоимость продукции на годы прогнозного периода с точностью в несколько процентов, что в принципе недостижимо. Ведь даже менеджеры высшего звена средних и крупных предприятий в большинстве случаев не могут с уверенностью спрогнозировать даже на ближайший год цену на выпускаемую продукцию своего предприятия.

Б.Е. Лужанский [19] путем имитационных экспериментов на компьютере методом Монте-Карло исследовал точность метода дисконтированных денежных потоков на конкретном примере рыночной оценки предприятия при реальном изменении случайных и неопределенных факторов - исходной информации и внутренних параметров модели, значения которых определяются оценщиком. Таким образом, стоимости дисконтированных денежных потоков в этих экспериментах при изменении показателей в реальном диапазоне варьировались от 10 до 230 млн долларов. То есть, при изменении параметров модели в «разумных» пределах верхняя «разумная», «доказуемая» с помощью метода ДДП стоимость предприятия отличалась от нижней также «разумной», «доказуемой» стоимости в 23 раза. Все эти эксперименты убедительно иллюстрируют нулевую ценность метода дисконтирования денежных потоков для объективной оценки бизнеса предприятий и поистине его бесценность для «заказной» оценки. 
В работе В.С. Зимина и В.Н. Тришина приведены результаты приближенных расчетов стоимостей пакетов акций четырех российских предприятий с учетом новых данных, ранее оцененных, методом ДДП ведущими мировыми аудиторскими компаниями [20]. Нами были перенесены модели двух из четырех этих бумажных отчетов в MS Excel и полностью пересчитаны как при имеющихся на тот момент прогнозах по цене продукции на момент оценки, так и при реально осуществившихся за прошедшие годы, а также новых на сегодняшний день прогнозах за оставшиеся годы прогнозного периода. Проведенные авторами расчеты показали, что стоимости пакетов акций этих двух из четырех компаний на самом деле выше в 5-6 раз. Заметим, что отчеты были выполнены по существующим меркам на высоком уровне, а столь значительная ошибка в определении рыночной стоимости предприятий, по нашему мнению, явилась следствием того, что при первоначальном дисконтировании были учтены результаты долгосрочного прогнозирования. Ценовая конъюнктура рынка за длительный период значительно изменилась в условиях неопределенности рыночной среды. Данные расчеты демонстрируют большую зависимость метода ДДП от изменений прогнозируемой стоимости продукции предприятий и невозможность с его помощью объективно и адекватно оценивать инвестиционную привлекательность компании на долгосрочную перспективу.

\section{Заключение}

Таким образом, основываясь на результатах исследования, нами предложены следующие методы по улучшению качества оценки привлекательности бизнеса с использованием метода ДДП в целях повышения общей инвестиционной привлекательности российского бизнеса не только в АТР, но и во всем мировом сообществе.

1. Из оценочной практики и стандартов оценки должны быть исключены методы (модели) оценки предприятий, предоставляющие оценщику возможность произвольно назначать стоимость предприятия в слишком широком возможном диапазоне.

2. Модель не должна быть логически противоречива, для нее должны быть выбрана соответствующая область (области) применения. Влияние параметров модели, зависящих от отдаленного будущего, должно в «разумной» модели быть сведено к минимуму.

3. Метод ДДП целесообразно использовать в оценке привлекательности средних и крупных предприятий, но только после его модификации, уменьшающей влияние ошибки прогнозирования к концу прогнозного периода, а также терминальной стоимости. Вариантов модификаций может быть предложено много, а какая из них более адекватна реальности, должна показать практика расчетов.

4. Итоговой раздел о выборе стоимости должен занимать не одну страницу, как в сегодняшних отчетах, а содержать подробный анализ оценщиком деятельности предприятия, его предположения, сомнения, логические выводы и обоснование решения о выборе окончательной стоимости. Заказчик, в свою очередь, вправе выбирать, согласиться ему с логикой оценщика или нет. 
5. Для защиты от «неправильной» оценки покупатель и продавец могут прописать будущие финансовые показатели бизнеса (выручку, валовую прибыль, EBITDA, FCF), и в случае неисполнения плана бюджета (либо его существенного перевыполнения), продавец или покупатель примут на себя обязательства возместить ущерб другой стороне.

Подводя итог, следует сказать, что оценочные стандарты в России должны быть радикально обновлены при участии обновленного состава Экспертно-консультативного Совета при Министерстве экономического развития Российской Федерации, который и разрабатывает эти самые стандарты при активном участии представителей саморегулируемых организаций оценщиков. Предложенные авторами методы помогут улучшить качество оценки инвестиционной привлекательности российского бизнеса для потенциальных инвесторов.

\section{Список источников / References}

1. Дамодаран А. Инвестиционная оценка: Инструменты и методы оценки любых активов. Москва, Альпина Бизнес Букс, 2017. 1340 с. [Damodaran A. Investicionnaya ocenka: Instrumenty` i metody` ocenki lyuby`x aktivov [Investment Appraisal: Tools and Methods for Appraising Any Assets]. Moscow, Alpina Business Books Publ., 2017. 1340 p.]

2. Кудина М.В. Инвестиционная активность компании как фактор повышения ее стоимости. Экономические науки, 2018, №10, с. 201. [Kudina M.V. Investicionnaya aktivnost` kompanii kak faktor povy`sheniya ee stoimosti [Investment activity of the company as a factor in increasing its value]. E`konomicheskie nauki = Economic Sciences, 2018, no. 10, p. 201.]

3. Лукасевич И.Я. Финансовый менеджмент. Москва, Эксмо, 2016. 768 с. [Lukasevich I.Ya. Finansovy`j menedzhment [Financial management]. Moscow, ECSMO Publ., 2016, 768 p.]

4. Чеботарев Н.Ф. Оценка стоимости предприятия (бизнеса). Москва, ИТК «Дашков и Ко», 2015. 253 c. [Chebotarev N.F. Ocenka stoimosti predpriyatiya (biznesa) [Valuation of the enterprise (business)]. Moscow, ITK “Dashkov \& Co" Publ., 2015. 253 p.]

5. Шипов В.Н. Специфика оценки стоимости предприятий в условиях современной российской экономики. Дайджест-Финансы, 2015. №9 (69), сс. 19-32. [Shipov V.N. Specifika ocenki stoimosti predpriyatij v usloviyax sovremennoj rossijskoj e'konomiki [The specifics of assessing the value of enterprises in the modern Russian economy]. Dajdzhest-Finansy` = Digest Finance, 2015, no. 9 (69), pp. 19-32.]

6. Щербаков В.А., Щербакова Н.А. Оценка стоимости предприятия (бизнеса). Москва, Омега-Л, 2016. 288 с. [Shherbakov V.A., Shherbakova N.A. Ocenka stoimosti predpriyatiya (biznesa) [Valuation of the enterprise (business)]. Moscow, Omega-L Publ., 2016. 288 p.]

7. Виленский П.Л., Лившиц В.Н., Смоляк С.А. Оценка эффективности инвестиционных проектов. Москва, АPMO, 2017. 888 с. [Vilenskij P.L., Livshicz V.N., Smolyak S.A. Ocenka e'ffektivnosti investicionny`x proektov [Evaluation of the effectiveness of investment projects]. Moscow, ARMO Publ., 2017. 888 p.]

8. Липсиц И.В. Инвестиционный анализ. Москва, ИНФРА-М, 2015. 320 с. [Lipsicz I.V. Investicionny`j analiz [Investment analysis]. Moscow, INFRA-M Publ., 2015. 320 p.]

9. Попов В.М. Бизнес-планирование. Москва, Финансы и статистика, 2016. 16 с. [Роpov V.M. Biznes-planirovanie [Business planning]. Moscow, Finance and statistics, 
2016. 16 р.]

10. Сидорович А.В. Курс экономической теории: Общие основы экономической теории. Микроэкономика. Макроэкономика. Основы национальной экономики. Москва, Дело и Сервис, 2018. 1035 с. [Sidorovich A.V. Kurs e`konomicheskoj teorii: Obshhie osnovy` e`konomicheskoj teorii. Mikroe`konomika. Makroe`konomika. Osnovy`nacional`noj e`konomiki [Economic Theory Course: General Foundations of Economic Theory. Microeconomics. Macroeconomics. Fundamentals of the National Economy]. Moscow, Business and Service, 2018. 1035 p.]

11. Филин С.А. Управление инвестициями в инновационной сфере экономики в условиях риска и неопределенности. Москва, ЮНИЦ, 2015. 73 с. [Filin S.A. Upravlenie investiciyami $\mathrm{v}$ innovacionnoj sfere e`konomiki $\mathrm{v}$ usloviyax riska i neopredelennosti [Investment Management in the Innovative Sphere of the Economy under Risk and Uncertainty]. Moscow, YuNITZ Publ., 2015. 73 p.]

12. Zhitlukhina O.G., Rakutko S.Yu., Berezhnova E.I., Selezneva E.Yu., Belik E.V., Shalaeva N.I., Denisevich E.I., Belik N.V., Saenko Z.E., Sultanova A.A. Issues of Falsifying Financial Statements in terms of Economic Security. International Journal of Environmental \& Science Education, 2016, vol. 11, issue 17, pp. 10163-10176.

13. Смоляк С.А. Дисконтирование денежных потоков в задачах оценки эффективных инвестиций. Москва, Эксмо, 2015. 324 с. [Smolyak S.A. Diskontirovanie denezhny`х potokov $v$ zadachax ocenki e`ffektivny`x investicij [Discounting cash flows in the tasks of evaluating effective investments]. Moscow, ECSMO Publ., 2015. 324 p.]

14. Масленникова О.П. Методические рекомендации по оценке эффективности инвестиционных проектов. Москва, ИНФРА-М, 2016. 186 с. [Maslennikova O.P. Metodicheskie rekomendacii po ocenke e`fektivnosti investicionny`x proektov [Methodological recommendations for assessing the effectiveness of investment projects]. Moscow, INFRA-M Publ., 2016. 186 p.]

15. Фомин Е.П. Дисконтирование денежного потока в оценке фирмы. Экономическая теория, 2016. 81 c. [Fomin E.P. Diskontirovanie denezhnogo potoka v ocenke firmy' [Discounting cash flow in company valuation]. Economic theory, 2016. 81 p.]

16. Кривелевич М.Е., Лазарев В.А. Финансовая трансформация в АТР: разработка вариантов российского участия. Азиатско-Тихоокеанский регион: экономика, политика, право, 2014, №1-2, сc. 9-19. [Krivelevich M.E., Lazarev V.A. Finansovaya transformaciya v ATR: razrabotka variantov rossijskogo uchastiya [Financial Transformation in the Asia-Pacific Region: Developing Options for Russian Participation]. Aziatsko-Tixookeanskij region: e konomika, politika, parvo = Asia-Pacific region: economics, politics, law, 2014, no. 1-2, pp. 9-19.]

17. Zhitlukhina O.G., Babak L.N., Rakutko S.Y., Selezneva E.Y., Denisevich E.I., Berezhnova E.I., Belik E.V., Khegay E.V., Gubareva O.I., Mikhalyova O.L. Specificity of the relationship between project management and organizational culture. Journal of Entrepreneurship Education, 2018, vol. 21, issue 3. Available at: https://www.abacademies.org/articles/specificity-of-the-relationship-between-projectmanagement-and-organizational-7211.html

19. Лопатников А. Единые стандарты профессиональной практики оценки. Москва, APMO, 2016. [Lopatnikov A. Ediny`e standarty` professional`noj praktiki ocenki [Uniform standards for professional assessment practice]. Moscow, ARMO Publ., 2016.]

19. Лужанский Б.Е. Оценка стоимости с учетом погрешности и неопределенности исходной информации. Доходный подход. Москва, 2006. [Luzhanskij B.E. Ocenka stoimosti s uchetom pogreshnosti i neopredelennosti isxodnoj informacii. Doxodny j podxod [Estimating the cost taking into account the error and uncertainty of the initial information. Income approach]. Moscow, 2006.]

20. Тришин В.Н., Зимин В.С. Прогнозирование и анализ точности метода дисконти- 
рованных денежных потоков. Имущественные отношения в Российской Федераuุuи, 2016, №7 (185), сc. 27-35. [Trishin V.N., Zimin V.S. Prognozirovanie i analiz tochnosti metoda diskontirovanny`x denezhny`x potokov [Forecasting and analysis of the accuracy of the discounted cash flow method]. Imushhestvenny'e otnosheniya $v$ Rossijskoj Federacii = Property relations in the Russian Federation, 2016, no. 7 (185), pp. 27-35.]

\section{Сведения об авторах / About authors}

Скалкин Дмитрий Владимирович, магистрант, Школа экономики и менеджмента, Дальневосточный федеральный университет. 690922 Россия, г. Владивосток, о-в Русский, кампус ДВФУ, корпус G. E-mail: skalkin.dv@ students.dvfu.ru

Dmitriy V. Skalkin, Master Student, School of Economics and Management, Far Eastern Federal University. Bldg. G, FEFU campus, Russky Island, Vladivostok, Russia, 690922.

E-mail: skalkin.dv@students.dvfu.ru

Селезнева Елена Юрьевна, кандидат исторических наук, доцент кафедры бухгалтерского учета, анализа и аудита, Школа экономики и менеджмента, Дальневосточный федеральный университет. 690922 Россия, г. Владивосток, о-в Русский, кампус ДВФУ, корпус G.

E-mail: selezneva.eyu@dvfu.ru

Elena Y. Selezneva, Candidate of Historical Sciences, Associate Professor, Department of Accounting, Analysis and Audit, School of Economics and Management, Far Eastern Federal University. Bldg. G, FEFU campus, Russky Island, Vladivostok, Russia, 690922. E-mail: selezneva.eyu@dvfu.ru

Ракутько Светлана Юрьевна, кандидат экономических наук, доцент, доцент кафедры бухгалтерского учета, анализа и аудита, Школа экономики и менеджмента, Дальневосточный федеральный университет.. 690922 Россия, г. Владивосток, о-в Русский, кампус ДВФУ, корпус G. E-mail: rakutko.syu@dvfu.ru

Svetlana Y. Rakutko, Candidate of Economical Sciences, Associate Professor of the Department of Accounting, Analysis and Audit, School of Economics and Management, Far Eastern Federal University. Bldg. G, FEFU campus, Russky Island, Vladivostok, Russia, 690922.

E-mail: rakutko.syu@dvfu.ru 